\title{
A note on simultaneous approximation on Vitushkin sets
}

\author{
Raymond MORTINI and Rudolf RupP \\ (Received February 5, 2020) \\ (Revised May 13, 2020)
}

\begin{abstract}
Given a planar Jordan domain $G$ with rectifiable boundary, it is well known that smooth functions on the closure of $G$ do not always admit smooth extensions to $\mathbb{C}$. Further conditions on the boundary are necessary to guarantee such extensions. On the other hand, Weierstrass' approximation theorem yields polynomials converging uniformly to $f \in C(\bar{G}, \mathbb{C})$. In this note we show that for Vitushkin sets $K$ with $K=\overline{K^{\circ}}$ it is always possible to uniformly approximate on $K$ the smooth function $f \in C^{1}(K, \mathbb{C})$ by smooth functions $f_{n}$ in $\mathbb{C}$ so that also $\bar{\partial} f_{n}$ converges uniformly to $\bar{\partial} f$ on $K$. As a byproduct we deduce from its "smooth in a neighborhood version" the general Gauss integral theorem for functions whose partial derivatives in $G$ merely admit continuous extensions to its boundary.
\end{abstract}

\section{Introduction}

Let $K$ be a compact set in $\mathbb{C}$ satisfying $\overline{K^{\circ}}=K$. Given a function $f \in$ $C(K, \mathbb{C}) \cap C^{1}\left(K^{\circ}, \mathbb{C}\right)$, suppose that the partial derivatives, $f_{x}$ and $f_{y}$, or equivalently the Wirtinger derivatives $\bar{\partial} f$ and $\partial f$, admit continuous extensions to $\overline{K^{\circ}}=K$. We denote this set of functions by $C^{1}(K, \mathbb{C})$. Is $f$ the trace of a $C^{1}$-function in $\mathbb{C}$ ? This very classical subject has been considered and generalized by generations of mathematicians. Let us only mention the theories developed by Hassler Whitney and later by Charles Fefferman. It is very easy to come up with a counterexemple.

As soon as there are cusps, these extensions are not possible (see Figure 1). In fact such a function, $F$, would locally satisfy a Lipschitz condition. In particular, around small disks centered at the origin, we would have for $x<0$

$$
x^{2}=\left|F\left(x, x^{3}\right)-F\left(x,-x^{3}\right)\right| \leq L\left\|\left(x, x^{3}\right)-\left(x,-x^{3}\right)\right\|=2 L\left|x^{3}\right| .
$$

Thus, dividing by $x^{2}$, we arrive at $1 \leq 2 L|x|$, which is a contradiction whenever $|x|$ is small.

2010 Mathematics Subject Classification. Primary 30E10; Secondary 26B20.

Key words and phrases. Vitushkin sets, uniform approximation, smooth functions. 


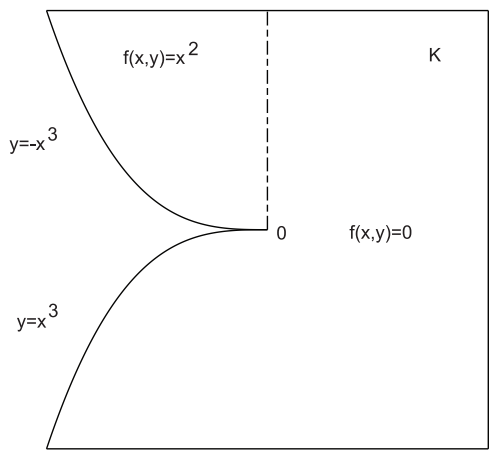

Fig. 1. Not the trace of a $C^{1}$-function

For smooth domains, though, smooth extensions are possible [8]:

TheOrem 1 (Seeley). Let $D \subseteq \mathbb{C}$ be a bounded domain with $C^{k}$-boundary. Then every $f \in C^{k}(\bar{D})$ is the trace on $\bar{D}$ of a function $F \in C_{c}^{k}(\mathbb{C})$, where $C_{c}^{k}(\mathbb{C})$ is the space of $k$-times continuously differentiable functions with compact support in $\mathbb{C}$.

See also [5, p. 69] for a sketched proof. For example, if $k=1$ and $D=\mathbb{D}$, then a $C^{1}$-extension to $\mathbb{R} \times[0,3 / 2]$ of $p$, defined by $p(\theta, r)=f\left(r e^{i \theta}\right)$ for $(\theta, r) \in \mathbb{R} \times[0,1[$, is given by

$$
F(\theta, r)= \begin{cases}p(\theta, r) & \text { if } 0 \leq r \leq 1 \\ n(\theta, r):=3 p(\theta, 2-r)-2 p(\theta, 3-2 r) & \text { if } 1 \leq r \leq 3 / 2\end{cases}
$$

Multiplying $F$ with a $C^{\infty}$-function that is independent of $\theta$, equals 1 on $\mathbb{R} \times[0,6 / 5]$ and 0 on $\mathbb{R} \times\left[7 / 5, \infty\left[\right.\right.$ now yields a $C_{c}^{1}\left(\mathbb{R}^{2}\right)$ extension of $p$. Denote this by $F$ again. Now by using the $2 \pi$-periodicity of $F$, the function $f^{*}: \mathbb{R}^{2} \backslash\{(0,0)\} \rightarrow \mathbb{C}$, given by

$$
f^{*}(x, y)=F(\theta, r) \quad \text { where } x=r \cos \theta, y=r \sin \theta, r>0,-\pi \leq \theta<\pi,
$$

is well-defined and so a $C^{1}$-extension of $f: \mathbb{D} \rightarrow \mathbb{C}$.

So, as smooth extensions are not always possible, one may ask whether a simultaneous approximation of $f \in C^{1}(K, \mathbb{C})$ and its derivatives is possible. As a contribution to an answer to this question we will show that for a wide class of compacta it is always possible to find $f_{n} \in C^{1}(\mathbb{C}, \mathbb{C})$ such that $f_{n}$ converges uniformly on $K$ to $f$ and $\bar{\partial} f_{n}$ to $\bar{\partial} f$. These compacta arise in the theory of rational approximation. They are the so called Vitushkin sets (see below). 


\section{Simultaneous approximation}

Recall that $A(K)$ is the space of all functions continuous on the compact set $K \subseteq \mathbb{C}$ and holomorphic in its interior $K^{\circ}$, and that $R(K)$ is the uniform closure on $K$ of the set of rational functions without poles on $K$. The following definition is motivated by a very deep result of A. G. Vitushkin, who characterized those planar compacta $K$ for which $A(K)=R(K)$ (see [6, p. 217]).

Definition 1. A compact set $K$ in $\mathbb{C}$ is called a Vitushkin set if $R(K)=$ $A(K)$.

For example, if $K \subseteq \mathbb{C}$ is a compact set such that $\mathbb{C} \backslash K$ has only finitely many connected components, then $A(K)=R(K)$ (see [6]). For $f \in C^{1}(K, \mathbb{C})$, we denote the extensions of $f_{x}$ and $f_{y}$ from $K^{\circ}$ to $\overline{K^{\circ}}$ by $f_{x}^{e}$ and $f_{y}^{e}$, respectively. Moreover, $\partial_{e} f$ denotes the continuous extension of $\partial f:=\left(f_{x}-i f_{y}\right) / 2$ to $\overline{K^{\circ}}$. Note that $\partial_{e} f=\left(f_{x}^{e}-i f_{y}^{e}\right) / 2$ due to the uniqueness of these extensions. The same applies for $\bar{\partial}_{e} f$. Finally, let $\|f\|_{K}:=\sup \{|f(z)|: z \in K\}$. Here is now our result.

THEOREM 2. Let $K \subseteq \mathbb{C}$ be a Vitushkin set satisfying $K=\overline{K^{\circ}}$. Then, for every $f \in C^{1}(K, \mathbb{C})$, there exist $f_{n} \in C_{c}^{\infty}(\mathbb{C})$ such that

$$
\left\|f_{n}-f\right\|_{K} \rightarrow 0 \quad \text { and } \quad\left\|\bar{\partial} f_{n}-\bar{\partial}_{e} f\right\|_{K} \rightarrow 0 .
$$

Proof. Let

$$
F_{0}(z):=-\frac{1}{\pi} \int_{K} \frac{\bar{\partial}_{e} f(\zeta)}{\zeta-z} d \sigma_{2}(\zeta)
$$

where $\sigma_{2}$ denotes planar Lebesgue measure in $\mathbb{C}$. Since $F_{0}$ and $f \in C(K, \mathbb{C})$ and since $\bar{\partial} F_{0}=\bar{\partial} f$ on $K^{\circ}$ (see [2] and [7]), $F_{0}=f+h$ for some holomorphic function $h \in A(K)$. Fix a compact neighborhood $C$ of $K$ and consider Tietze extensions $h^{*}$ of $h$ to $C$ and $\bar{\partial}_{e} f$ of $\bar{\partial} f \in C\left(K^{\circ}, \mathbb{C}\right)$ to $C$. Since by hypothesis $A(K)=R(K)$, given $\varepsilon>0$, there is a rational function $W$ such that $\|W-h\|_{K}$ $<\varepsilon$. Let the open set $U$ be chosen so that

$$
K \subseteq U \subseteq C, \quad\left\|W-h^{*}\right\|_{\bar{U}}<\varepsilon .
$$

Now let $U_{n}$ be open neighboorhoods of $K$ with

$$
K \subseteq U_{n} \subseteq \overline{U_{n}} \subseteq U, \quad \overline{U_{n+1}} \subseteq U_{n}, \quad \bigcap_{n=1}^{\infty} U_{n}=K .
$$


By Weierstrass' approximation Theorem, there exist polynomials $W_{n} \in \mathbb{C}[x, y]$ so that

$$
\left\|\bar{\partial}_{e} f-W_{n}\right\|_{C}<1 / n
$$

Consider the Cauchy transforms

$$
F_{n}(z):=-\frac{1}{\pi} \int_{\bar{U}_{n}} \frac{W_{n}(\zeta)}{\zeta-z} d \sigma_{2}(\zeta),
$$

which are continuous on $\mathbb{C}$, and on $C$ let $f_{n}^{*}:=F_{n}-W$. Since $U_{n} \subseteq U$, we conclude that $f_{n}^{*} \in C^{\infty}\left(U_{n}\right)$. Due to the fact (see [1, p. 150]) that for all $z \in \mathbb{C}$

$$
\int_{K} \frac{1}{|\zeta-z|} d \sigma_{2}(\zeta) \leq 2 \sqrt{\pi \sigma_{2}(K)}
$$

we obtain on $K$ the following estimates:

$$
\begin{aligned}
\left|f-f_{n}^{*}\right| & =\left|\left(F_{0}-h\right)-\left(F_{n}-W\right)\right|=\left|\left(F_{0}-F_{n}\right)-(h-W)\right| \\
& \leq\left|-\frac{1}{\pi} \int_{K} \frac{\bar{\partial}_{e} f(\zeta)}{\zeta-z} d \sigma_{2}(\zeta)+\frac{1}{\pi} \int_{\bar{U}_{n}} \frac{W_{n}(\zeta)}{\zeta-z} d \sigma_{2}(\zeta)\right|+\varepsilon \\
& \leq \frac{1}{\pi} \int_{K} \frac{\left|\bar{\partial}_{e} f(\zeta)-W_{n}(\zeta)\right|}{|\zeta-z|} d \sigma_{2}(\zeta)+\frac{1}{\pi} \int_{\bar{U}_{n} \backslash K} \frac{\left|W_{n}(\zeta)\right|}{|\zeta-z|} d \sigma_{2}(\zeta)+\varepsilon \\
& \stackrel{(1.1)}{\leq} \frac{1}{n} \frac{2}{\sqrt{\pi}} \sqrt{\sigma_{2}(K)}+\underbrace{\left\|W_{n}\right\|_{C}}_{(1.1)} \frac{2}{\sqrt{\pi}} \underbrace{\sqrt{\sigma_{2}\left(\bar{U}_{n} \backslash K\right)}}_{\rightarrow 0 \text { as } n \rightarrow \infty}+\varepsilon
\end{aligned}
$$

$\leq 3 \varepsilon \quad$ for all $n \geq n_{0}$.

Moreover, on $U_{n}{ }^{1}$

$$
\begin{aligned}
\left|\bar{\partial}_{e} f-\bar{\partial} f_{n}^{*}\right| & =\left|\bar{\partial}_{e} f-\bar{\partial} F_{n}+\bar{\partial} W\right|=|\bar{\partial}_{e} f-W_{n}+\underbrace{\bar{\partial} W}_{=0}| \\
& =\left|\bar{\partial}_{e} f-W_{n}\right| \underset{(1.1)}{\leq 1 / n .}
\end{aligned}
$$

Hence $\left\|\bar{\partial}_{e} f-\bar{\partial} f_{n}^{*}\right\|_{K} \leq 1 / n$. The final approximating function $f_{n}$ now will be a cutoff of $f_{n}^{*}$. That is, let $O_{n}$ and $V_{n}$ be open sets with

$$
K \subseteq O_{n} \subseteq \bar{O}_{n} \subseteq V_{n} \subseteq \bar{V}_{n} \subseteq U_{n}
$$

\footnotetext{
${ }^{1}$ It is only here that we need $K$ to be a Vitushkin set, in order to obtain an approximating function $W$ with $\bar{\partial} W=0$.
} 
Choose $\alpha_{n} \in C_{c}^{\infty}(\mathbb{C})$ with $0 \leq \alpha \leq 1, \alpha_{n}=1$ on $O_{n}$ and $\alpha_{n}=0$ on $\mathbb{C} \backslash V_{n}$. Define $f_{n}$ by $f_{n}:=f_{n}^{*} \alpha_{n}$ on $V_{n}$ and $f_{n}:=0$ on $\mathbb{C} \backslash V_{n}$. Then it is easy to see that $f_{n} \in C_{c}^{\infty}(\mathbb{C}, \mathbb{C})$. Moreover, on $O_{n}$ we have $f_{n}=f_{n}^{*}$ as well as $\bar{\partial} f_{n}=\bar{\partial} f_{n}^{*}$, because

$$
\bar{\partial}\left(f_{n}^{*} \alpha_{n}\right)=\left(\bar{\partial} f_{n}^{*}\right) \underbrace{\alpha_{n}}_{=1}+f_{n}^{*} \underbrace{\bar{\partial} \alpha_{n}}_{=0}=\bar{\partial} f_{n}^{*} .
$$

Hence $f_{n}$ satisfies

$$
\left\|f_{n}-f\right\|_{K} \rightarrow 0 \quad \text { and } \quad\left\|\bar{\partial} f_{n}-\bar{\partial}_{e} f\right\|_{K} \rightarrow 0 .
$$

This finishes the proof of Theorem 2.

Here we have two questions: Is it possible to achieve also the uniform convergence of $\partial f_{n}$ to $\partial_{e} f$ ? And in how far the condition $K$ a Vitushkin set, can be relaxed?

\section{Gauss' Theorem revisited}

In [3] Carmona and Cufi gave a really magnificent proof of the complex version of the "smooth in a neighborhood version" of Gauss' theorem by using the $\bar{\partial}$-calculus, a proof that should be included in every complex analysis course (see [2, p. 213]). Recall that, by definition, the boundary of an admissible domain splits into finitely many pairwise disjoint $C^{1}$-Jordan curves with the appropriate orientation. We denote here the area integral with a double integral.

ThEOREM 3 (Gauss' Theorem, complex version). Let $G$ be an admissible domain and $U$ an open neighborhood of $\bar{G}$. Suppose that $f \in C^{1}(U)$. Then

$$
\int_{\partial G} f(z) d z=2 i \iint_{G} \bar{\partial} f(\zeta) d \sigma_{2}(\zeta) .
$$

The more general version for functions whose partial derivatives in $G$ merely admit continuous extensions to its boundary (denoted by $f \in C^{1}(\bar{G})$ ) can be shown in various ways. An obvious approach is to approximate the boundary curves of $G$ by curves $\gamma_{n}$ of the same type entirely contained in $G$ and such that

$$
\int_{\gamma_{n}} f(z) d z \rightarrow \int_{\partial G} f(z) d z
$$

(not so easy and rather technical). Another approach is given in [3] by using a result in advanced function theory that the derivative of a Riemann map 
$r: \mathbb{D} \rightarrow \Omega$ onto a Jordan domain belongs to the Hardy space $H^{1}$ if and only if the boundary of $\Omega$ is rectifiable (see [4, p. 44]).

We now apply Theorem 2 in the special case where $K=\bar{D}$ for some admissible domain $D$, to deduce the following general version of Gauss' Theorem from its special version 3 .

THeOrem 4 (Gauss). Let $D$ be an admissible domain and $f \in C^{1}(\bar{D})$. Then

$$
\int_{\partial D} f(z) d z=2 i \iint_{D} \bar{\partial} f(\zeta) d \sigma_{2}(\zeta)
$$

Proof. Let $K=\bar{D}$. Since $D$ is admissible, $K$ has only finitely many holes. Hence, by Mergelyan's Theorem for rational approximation [6], $R(K)=$ $A(K)$. Thus $K$ is a Vitushkin set. According to Theorem 2, choose $f_{n} \in$ $C_{c}^{\infty}(\mathbb{C})$ such that

$$
\left\|f_{n}-f\right\|_{K} \rightarrow 0 \quad \text { and } \quad\left\|\bar{\partial} f_{n}-\bar{\partial}_{e} f\right\|_{K} \rightarrow 0 .
$$

By Theorem 3,

$$
\int_{\partial D} f_{n}(z) d z=2 i \iint_{D} \bar{\partial} f_{n}(\zeta) d \sigma_{2}(\zeta) .
$$

The uniform convergence of $f_{n}$ to $f$ and $\bar{\partial} f_{n}$ to $\bar{\partial}_{e} f$ on $K$ now yields the assertion.

\section{Acknowledgements}

We thank Peter Pflug (Oldenburg, Germany) for providing reference [5] and Jochen Wengenroth (Trier, Germany) for useful comments and reference [8] in connection with smooth extensions of smooth functions.

\section{References}

[1] A. Browder, Introduction to Function Algebras, W. A. Benjamin, Inc. New York, (1969).

[2] J. Bruna, J, Cufi, Complex Analysis, EMS Textbooks in Math., European Math. Soc., Zürich, 2013.

[3] J. Carmona, J. Cufi, The index of a plane curve and Green's formula, Rend. del. Circolo Mat. di Palermo, Ser. II, 53 (2004), 103-128.

[4] P. L. Duren, Theory of $H^{p}$-spaces, Academic Press, New York, 1970.

[5] W. Fischer, I. Lieb, Ausgewählte Kapitel aus der Funktionentheorie, Vieweg, Braunschweig, 1988.

[6] T. W. Gamelin, Uniform algebras, Chelsea, New York, 1984. 
[7] R. Mortini, R. Rupp, A solution to the Bézout equation in $A(K)$ without Gelfand theory, Archiv Math. 99 (2012), 49-59.

[8 8 R. T. Seeley, Extension of $C^{\infty}$ functions defined in a half space, Proc. Amer. Math. Soc. 15 (1964), 625-626.

\author{
Raymond Mortini \\ Université de Lorraine \\ Département de Mathématiques et Institut Élie Cartan de Lorraine \\ UMR 7502, F-57073 Metz, France \\ E-mail: raymond.mortini@univ-lorraine.fr

\section{Rudolf Rupp} \\ Fakultät für Angewandte Mathematik, \\ Physik und Allgemeinwissenschaften \\ Technische Hochschule Nürnberg, Georg Simon Ohm \\ Kesslerplatz 12 \\ D-90489 Nürnberg, Germany \\ E-mail: rudolf.rupp@th-nuernberg.de
}

